\title{
Right Ventricular Structure and Function as Possible Determinants of Surgical Outcome 30 Years After Repair of Tetralogy of Fallot
}

\author{
Sonia F. Mesquita, Raquel Snitcowsky (in memoriam), Antonio Augusto Lopes
}

São Paulo, SP - Brazil

Objective - To identify the variables that may be involved in the persistence of symptoms (functional class II, III, or IV vs. I) in patients being followed up for 30 years after surgical repair of tetralogy of Fallot.

Methods - Fifty-three patients (27 women), who underwent corrective surgery for tetralogy of Fallot between 1960 and 1970, were studied. Their ages ranged from 7 months to 26 years. At the end of follow-up, 13 patients were asymptomatic and the remaining were in functional class II $(N=24), I I I(N=15)$, and IV $(N=1)$. To differentiate asymptomatic from symptomatic patients, the following variables were analyzed: age at surgery, need for widening the pulmonary ring and trunk, need for a second (2nd $O P)$ or 3 rd operation, residual defect of the interventricular septum, residual regurgitation of the pulmonary valve, systolic gradient through the right ventricular outflow tract, right ventricular dilation or hypertrophy $(R V H)$, cardiothoracic index (CTI), right and left ventricular ejection fraction ( $R V E F / L V E F)$, and arrhythmias.

Results - The univariate analysis showed an association between the presence of symptoms and the 2 nd $O P$ $(P=0.03)$, an increase in the $C T I(P=0.0001)$, moderate to severe $R V H(P=0.002)$, and dilation $(P=0.0003)$. In the logistic regression model, the combination of the $2 n d O P$ $(P=0.008)$, the RVH $(P=0.002)$, and the reduction in $R V E F(P=0.01)$ determined the presence of symptoms.

Conclusion - Despite the surgical treatment, right ventricular remodeling and performance were the major determinants in the late follow-up of tetralogy of Fallot.

Key words: tetralogy of Fallot, surgery, right ventricular structure

Instituto de Coração of the Hospital das Clínicas - FMUSP

Mailing address: Antonio Augusto Lopes - InCor - Cardiologia Pediátrica e Cardiopatias Congênitas - Av. Dr. Eneas C. Aguiar, 44

Cep 05403-000 - São Paulo, SP, Brazil - E-mail: aablopes@usp.br

English version by Stela Maris C. e Gandour

Received 7/1/02

Accepted 4/14/03
Currently, expectations of good surgical results in patients undergoing repair of tetralogy of Fallot exist. This perspective is mainly related to the evolution of surgical techniques and intensive postoperative measures, and the possibility of correction in an early phase in children and even in neonates. However, this favorable situation may not be the case for patients who underwent surgery 4 decades ago.

Postoperative complications have been reported and related, from the first moment, to the indication of reoperation $^{1,2}$, and, at a second moment, to the performance of surgical treatment in later phases with a consequent reduction in survival ${ }^{3}$ and in right ventricular remodeling ${ }^{4-6}$, justifying systolic and diastolic dysfunctions in late outcome. The present study analyzed the clinical features of patients undergoing repair of tetralogy of Fallot between 1960 and 1970 who remained alive 30 years or more after surgery.

Our major objective was to identify possible predictive variables that could help in differentiating between asymptomatic patients and those with persistent symptoms.

\section{Methods}

The study comprised patients being treated in the department of pediatric cardiology and congenital heart diseases in the adult at the Instituto do Coração between 1995 and 2001. All patients with at least 30 years of postoperative evolution were studied. Their medical records included systematic annual clinical evaluations during the period studied. The patients with pulmonary atresia and interventricular septal defect were excluded. For 4 patients, who had undergone reoperation, the date of the first operation was considered to be the beginning of the study (March 1960 November 1970). It is worth noting that many patients referred to the Instituto do Coração come from distant regions of the country, and social reasons made their follow-up difficult to continue. Therefore, the patients included in this study were not consecutive, and they were assumed to represent the population of patients operated on between 1960 and 1970 who were alive 30 years after the operation. 
The patients were assessed annually by the same medical team during the period of at least 30 years following surgical repair of tetralogy of Fallot. The patients underwent a complete series of noninvasive examinations at the time of the last clinical assessment. Data from the postoperative period longer than 30 years, including examinations and surgical reports, were obtained from the medical records of the patients.

Of the surgical information, age at surgery and the type of the procedure performed for right ventricular outflow tract correction were written down, mainly the need for widening the trunk of the pulmonary artery and its branches and the ring of the pulmonary valve. Clinical complications and the eventual need for a second or third operation for correction of the residual defects were also analyzed.

At the end of the follow-up, the following data were obtained: functional capacity according to the criteria of the New York Heart Association (NYHA), complaints of palpitations, and the occurrence of syncopes. In addition to conventional electrocardiography, electrocardiographic monitoring (24-hour Holter) was used to identify arrhythmias. The cardiothoracic index was calculated using chest radiography and right and left ventricular ejection fractions obtained on radioisotopic ventriculography. Doppler echocardiography was used to assess the residual intracardiac defects and to estimate the systolic gradient in the right ventricular outflow tract, the degree of regurgitation of the pulmonary and tricuspid valves, and the degree of hypertrophy and dilation of the right ventricle. The 2 latter variables were qualitatively assessed by 2 independent observers who ignored the other clinical data. The information was classified into categories: $0=$ absent or mild; $1=$ modera- te or severe. In cases where a consensus was not reached, a third opinion was obtained.

The results are shown as means \pm standard deviations, or as medians and their respective variation range. The variables were nonparametrically tested, using the chi-square test or the Fisher exact test. For the continuous variables, the Student $t$ test was used. The possible associations of the variables and the presence of symptoms were tested using, initially, univariate analysis (chi-square test and Fisher test or Student $t$ test), and, later, the multivariate analysis (logistic regression). The significance level of 0.05 was adopted in all tests.

\section{Results}

The study comprised 53 (27 women) patients followed up for a mean period of 32.5 (30-39.7) years, with a mean age at surgery of 6 years ( 7 months to 26 years). At the end of the follow-up, the patients' ages ranged from 31.2 to 60 years, and 13 patients were asymptomatic and 40 were in the following NYHA functional classes: II ( $\mathrm{N}=24)$, III ( $\mathrm{N}=15)$, and $\mathrm{IV}(\mathrm{N}=1)$.

The major symptoms reported by the patients were as follows: dyspnea on exertion in 40 patients, palpitations in 19, and syncope in 4. Thromboembolism and infective endocarditis were rare events in this group of patients ( 1 and 2 episodes, respectively). Cyanosis not related to residual intercavitary communication and associated with the development of pulmonary arteriovenous fistulas was observed in 2 patients.

Of the 19 patients complaining of palpitations, $18 \mathrm{had}$ relevant atrial or ventricular arrhythmias on the electrocar-

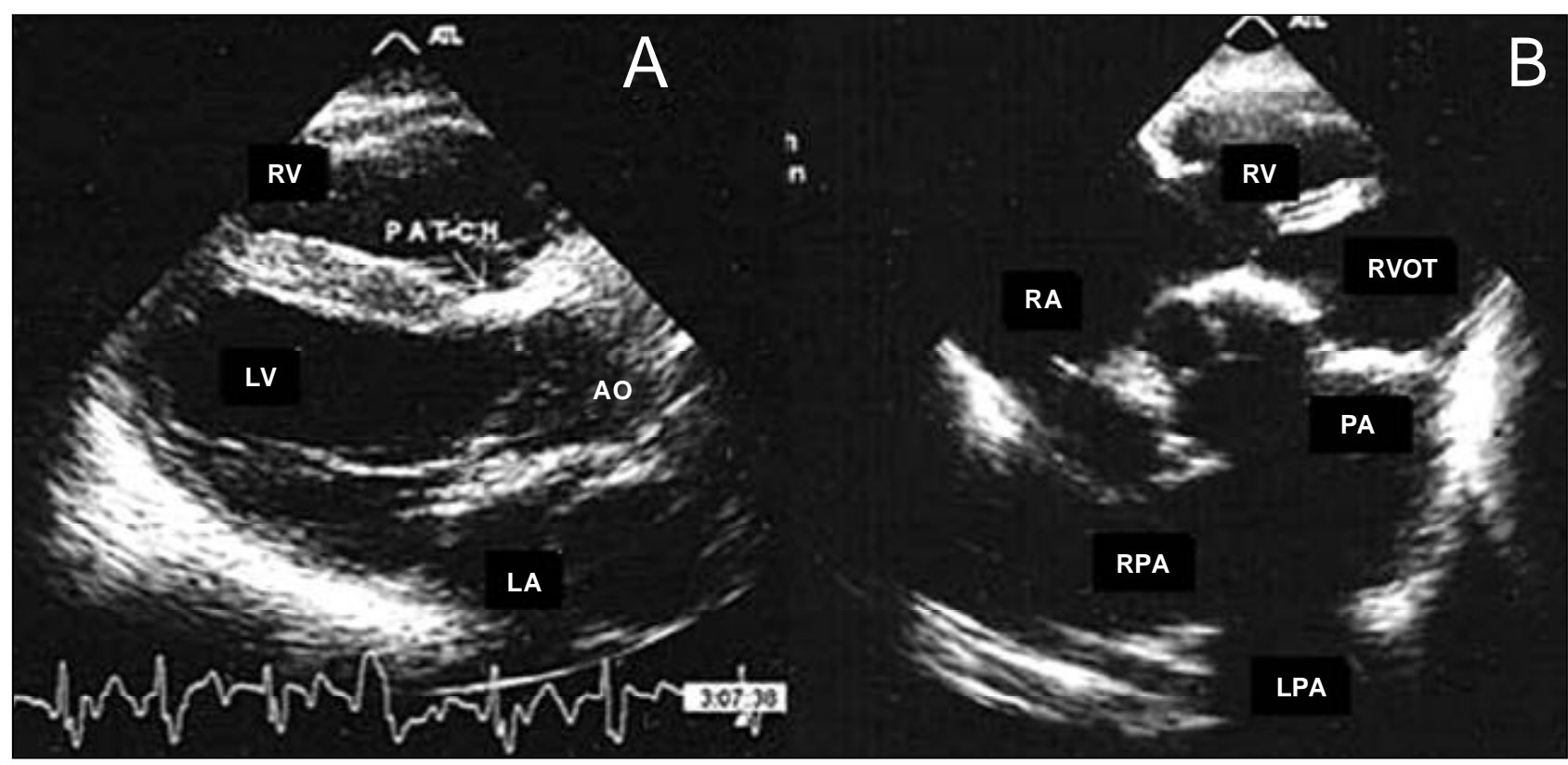

Fig. 1 - Echocardiographic analysis in the postoperative evolution of a 38.7-year-old man operated upon at the age of 10.9 years, with postoperative evolution of 49.5 years. The patient was reoperated upon for pulmonary valve replacement, tricuspid valve plasty, and closure of a residual interventricular septal defect. Although the results regarding the correction of RVOT (A, patch) were satisfactory, the patient remains symptomatic (FC III) with a CTI of 65 and RVEF of 27\%. LA- left atrium; LV-left ventricle; RA- right atrium; RV-right ventricle; PA- pulmonary trunk; RPA- right pulmonary artery; LPA- left pulmonary artery; RVOT- right ventricular outflow tract. 


\begin{tabular}{|c|c|c|c|c|}
\hline & $\begin{array}{l}\text { Cardiothoracic } \\
\text { index }(\%)\end{array}$ & $\begin{array}{l}\text { RV/PA gradient } \\
\text { (mmHg) }\end{array}$ & $\begin{array}{l}\text { RVEF } \\
(\%)\end{array}$ & $\begin{array}{c}\text { LVEF } \\
(\%)\end{array}$ \\
\hline $\begin{array}{l}\text { Mean } \pm \text { standard } \\
\text { deviation }\end{array}$ & $56 \pm 5$ & $30 \pm 20$ & $42 \pm 9$ & $51 \pm c$ \\
\hline Variation & $46-68$ & $4-114$ & $13-58$ & $28-69$ \\
\hline Median & 56 & 23 & 42 & 51 \\
\hline
\end{tabular}

diogram or on Holter (specificity of $95 \%, \mathrm{P}=0.0004$ ). However, the detection of the symptom was not sensitive enough to justify the arrhythmias detected, because that symptom was reported by only 18 of the 34 patients who had significant arrhythmias.

During follow-up, 26 patients underwent a second surgery, and 4 of them underwent a third. Reoperation was performed mainly to treat the residual obstruction of the right ventricular outflow tract $(\mathrm{N}=19)$, to close an interventricular septal defect $(\mathrm{N}=7)$, to close an interatrial septal defect $(\mathrm{N}=4)$, to replace the pulmonary valve $(\mathrm{N}=8)$, and to perform tricuspid valve plasty ( $\mathrm{N}=3$ ) (fig. 1).

At the end of follow-up, the most significant findings in regard to residual defects were interventricular septal defect $(\mathrm{N}=2)$; systolic gradient between the right ventricle and the pulmonary artery, ranging from 4 to $114 \mathrm{mmHg}$ in all patients; moderate to severe pulmonary $(\mathrm{N}=23)$ and tricuspid $(\mathrm{N}=7)$ regurgitation.

The values of the cardiothoracic index and of the right and left ventricular ejection fractions obtained at the end of follow-up are shown in table I. Moderate to severe hypertrophy and dilation were observed in 28 and 27 patients, respectively.

Aiming at identifying the factors that could be related to the presence of dyspnea on exertion in the late postoperative evolution, the following variables were analyzed: 1) age at surgery; 2) need for widening the ring of the pulmonary valve or the pulmonary artery; 3 ) need for a second or third operation; 4) residual systolic gradient in the right ventricular outflow tract; 5) moderate to severe pulmonary or tricuspid valve regurgitation; 6) residual interventricular septal defect; 7) cardiothoracic index; 8) moderate to severe right ventricular hypertrophy or dilation; 9) right and left ventricular ejection fraction; 10) presence of significant arrhythmias on Holter monitoring, such as sustained or nonsustained atrial tachycardia, nonsustained ventricular tachycardia, and the number of extrasystoles.

The univariate analysis showed a greater prevalence of dyspnea on exertion in the patients undergoing a second operation (88\%) as compared with those who were not reoperated upon $(63 \%)(\mathrm{P}=0.03)$. Compared with the asymptomatic patients, the symptomatic ones had greater cardiothoracic index values ( $52 \pm 2 \%$ and $57 \% \pm 4 \%$, respectively, $\mathrm{P}=0.0001$ ) and a greater prevalence of moderate to severe right ventricular hypertrophy ( $15 \%$ and $65 \%$, respectively, $\mathrm{P}=0.0002$ ) and dilation ( $8 \%$ and $65 \%$, respectively, $\mathrm{P}=0.0003$ ). The associa-

\begin{tabular}{|c|c|c|c|}
\hline Second operation & RVH & RVEF (\%) & Probability \\
\hline Yes & Yes & 35 & $99.9 \%$ \\
\hline No & Yes & 35 & $99.6 \%$ \\
\hline Yes & Yes & 45 & $99.7 \%$ \\
\hline Yes & No & 45 & $70.1 \%$ \\
\hline No & Yes & 45 & $95.1 \%$ \\
\hline No & No & 45 & $11.8 \%$ \\
\hline
\end{tabular}

tion of severe right ventricular hypertrophy and dilation was observed in 17 patients. However, no correlation was observed between right ventricular hypertrophy and the residual systolic gradient of the right ventricular outflow tract. However, the patients' age at surgery (with no direct relation to the symptoms) was significantly more expressive in patients who remained with a greater degree of right ventricular hypertrophy on late evolution $(10.1 \pm 7.5$ and $6.1 \pm 3$.8 years, respectively, $\mathrm{P}=0.01$ ).

Based on multivariate analysis (logistic regression), the model that better estimated the probability of the patient being symptomatic on late postoperative evolution was the one that included the second operation $(\mathrm{P}=0.008)$, right ventricular ejection fraction $(\mathrm{P}=0.01)$, and moderate to severe residual right ventricular hypertrophy $(\mathrm{P}=0.002)$ as independent variables. The regression model was as follows:

$$
\mathrm{P}=\frac{\mathrm{e}^{9.279+2.866(\text { nd OP) }-0.251(\mathrm{RVEF})+4.978(\mathrm{RVH})}}{1+\mathrm{e}^{9.279+2.866(2 \mathrm{nd} \mathrm{OP})-0.251(\mathrm{RVEF})+4.978(\mathrm{RVH})}}
$$

Where: the values of 2 ndOP and RVH are 0 or 1 , depending on the occurrence of a second operation and the presence of residual right ventricular hypertrophy, respectively; RVEF is the right ventricular ejection fraction in late follow-up; $\mathrm{P}$ is the estimated probability of a certain patient being symptomatic.

One potential use of the regression model is shown in table II, where the probability of having symptoms was estimated for arbitrary values of independent variables. Residual right ventricular hypertrophy appeared as an important determinant of persistent symptoms.

\section{Discussion}

Previous studies have focused on life expectancy and causes of death in the late postoperative evolution ${ }^{7,8}$. This study analyzed only the patients who remained alive 30 years after correction of tetralogy of Fallot, aiming at identifying the factors that could define the patients as asymptomatic or symptomatic. In the population studied, the age at surgery ranged from 7 months to 26 years, and the incidence of reoperations $(40 \%)$ for correction of residual obstruction in the right ventricular outflow tract was high. In our case series, altered right ventricular morphology and function were, on late evolution, factors related to a reduction in 
the patients' functional capacity. However, the major limitation of the present study was the impossibility of a quantitative analysis of the right ventricular hypertrophy and dilation variables.

Because this study includes only the survivors, the analysis of the arrhythmias as a cause of sudden death was not considered. As previously reported ${ }^{9-12}$, changes in cardiac rhythm and the detection of relevant arrhythmias were significant findings present even in the absence of palpitation.

An unexpected finding was that neither the magnitude of the residual gradient in the right ventricular outflow tract nor the presence of moderate to severe pulmonary valve regurgitation allowed differentiating between symptomatic and asymptomatic patients. This finding, however, should be carefully analyzed, because, in this study, neither information about the physical exercise capacity ${ }^{13-16}$ nor quantitative data about pulmonary valve regurgitation ${ }^{17}$ were available for most patients. On the other hand, the presence of marked right ventricular hypertrophy and dilation and the reduction in the right ventricular ejection fraction, closely related to dyspnea on exertion, were observed. These data allow us to believe that factors, such as residual lesions, are associated with symptoms only in the case of right ventricular remodeling or significant dysfunction, or both. Age at surgery did not directly correlate with the persistence of symptoms, but was closely associated with the development of right ventricular hypertrophy.

Another interesting finding was the influence of a second operation on the persistence of dyspnea on exertion. It is known that for many patients undergoing correction of tetralogy of Fallot in the 1960s at our institution, the attempts to relieve the obstruction of the right ventricular outflow tract involved infundibulectomy with careful resection of the muscle, but not opening of the ring of the pulmonary valve ${ }^{18,19}$. In fact, the transannular patch was performed in only 19 patients in this study. Consequently, the persistence of the obstruction of the right ventricular outflow tract was the major reason for referring the patients for reoperation. The need for a second operation may have been associated with myocardial damage consequent to the presence of residual lesions, which may explain the persistence of symptoms in these patients.

Finally, a right ventricular restrictive (vs. nonrestrictive) physiology, persisting long after the correction of tetralogy of Fallot, was reported to be associated with a less prolonged QRS, a lower risk of deleterious consequences of chronic pulmonary regurgitation, and a better tolerance to exercise ${ }^{15,20}$. Although apparently in disagreement with our findings of worse results in patients with significant right ventricular hypertrophy, this is not the case. In fact, in this study, hypertrophy was frequently associated with dilation, and, therefore, may have reflected a "remodeled" (and sometimes dysfunctional) ventricle and not a restrictive pattern, which has been associated with a better prognosis.

In conclusion, the long-term results after correction of tetralogy of Fallot, particularly in patients who underwent correction 30 to 40 years ago, seem to be more related to right ventricular remodeling and function than to residual defects. In this context, although excellent results with the early correction of tetralogy of Fallot currently exist, a referral for surgical treatment at a late age and residual lesions are factors of potential impact on right ventricular remodeling, becoming a concern in the case of some patients.

\section{References}

1. Lillehei CW, Varco RL, Cohen M, et al. The first open heart corrections of tetralogy of Fallot: a 26-31 year follow-up of 106 patients. Ann Surg 1986; 204:490-502.

2. Oechslin E, Harrison D, Harris L, et al. Reoperation in adults with repaired tetralogy of Fallot: indications and outcomes. In: Imai Y, Momma K. (Eds). Proceedings of $2^{\text {nd }}$ World Congress of PCCS 1997, p. 219-21.

3. Murphy JG, Gersh BJ, Mair DD, et al. Long-term outcome in patients undergoing surgical repair of tetralogy of Fallot. N Engl J Med 1993; 329:593-9.

4. Mitsuno M, Nakano S, Shimazaki Y, et al. Fate of right ventricular hypertrophy in tetralogy of Fallot after corrective surgery. Am J Cardiol 1993; 72:694-8.

5. Bishop JE, Rhodes S, Laurent GJ, Low RB, et al. Increased collagen synthesis and decreased collagen degradation in right ventricular hypertrophy induced by pressure overload. Cardiovasc Res 1994; 28:1581-5.

6. Seliem MA, Wu YT, Glenwright K. Relation between age at surgery and regression of right ventricular hypertrophy in tetralogy of Fallot. Pediatr Cardiol 1995; 16:53-5.

7. Norgaard MA, Lauridsen P, Helvind M, Pettersson G. Twenty-to-thirty-sevenyear follow-up after repair for Tetralogy of Fallot. Eur J Cardiothorac Surg 1999; 16:125-30.

8. Bacha EA, Sheule AM, Zurakowski D, et al. Long-term results after early primary repair of tetralogy of Fallot. J Thorac Cardiovasc Surg 2001; 122:154-61.

9. Downar E, Harris L, Kimber S, et al. Ventricular tachycardia after surgical repair to tetralogy of Fallot: results of intraoperative mapping studies. J Am Coll Cardiol 1992; 20:648-55.

10. Roos-Hesselink J, Perlroth MG, McGhie J, et al. Atrial arrhythmias in adults after repair to tetralogy of Fallot. Correlations with clinical, exercise, and echocardiographic findings. Circulation 1995; 91:2214-9.

11. Gatzoulis MA, Till JA, Somerville J, et al. Mechanoelectrical interaction in tetra- logy of Fallot: QRS prolongation relates to right ventricular size and predicts malignant ventricular arrhythmias and sudden death. Circulation 1995; 92:231-7.

12. Gatzoulis MA, Balaji S, Webber SA, et al. Risk factors for arrhythmia and sudden cardiac death late after repair of tetralogy of Fallot: a multicentre study. Lancet 2000; 356:975-81.

13. Grant GP, Garofano RP, Mansell AL, et al. Ventilatory response to exercise after intracardiac repair of tetralogy of Fallot. Am Rev Respir Dis 1992; 144:833-6.

14. Clark AL, Gatzoulis MA, Redington AN. Ventilatory responses to exercise in adults after repair of tetralogy of Fallot. Br Heart J 1995; 73:445-9.

15. Gatzoulis MA, Clark AL, Cullen S, et al. Right ventricular diastolic function 15 to 35 years after repair of tetralogy of Fallot: restrictive physiology predicts superior exercise performance. Circulation 1995; 91:1775-81.

16. Wessel HU, Paul MH. Exercise studies in tetralogy of Fallot: a review. Pediatr Cardiol 1999; 20:39-47.

17. Carvalho JS, Shinebourne EA, Busst C, et al. Exercise capacity after complete repair of tetralogy of Fallot: deleterious effects of residual pulmonary regurgitation. Br Heart J 1992; 67:470-3.

18. Zerbini EJ, Macruz R, Bittencourt D, et al. Total correction of complex of Fallo under extracorporeal circulation: immediate results in a group of 221 patients. J Thorac Cardiovasc Surg 1965; 49:430-9.

19. Zerbini EJ. The surgical treatment of the complex of Fallot: late results. J Thorac Cardiovasc Surg 1968; 58:158-77.

20. Norgard G, Gatzoulis MA, Moraes F, et al. Relationship between type of outflow tract repair and postoperative right ventricular diastolic physiology in tetralogy of Fallot: implications for long-term outcome. Circulation 1996; 94:3276-80. 